\title{
Diversidade de abelhas (Hymenoptera, Apidae) ao longo de um gradiente latitudinal na Mata Atlântica
}

\author{
Rodrigo Barbosa Gonçalves ${ }^{1,2}$ \& Carlos Roberto Ferreira Brandão ${ }^{1,3}$ \\ ${ }^{1}$ Museu de Zoologia da Universidade de São Paulo - USP, \\ Av. Nazaré, 481, CEP 04263-000, São Paulo, Brasil, e-mail: crfbrand@usp.br \\ ${ }^{2}$ Autor para correspondência: Rodrigo Barbosa Gonçalves, \\ e-mail: goncalvesrb@gmail.com, http://www.usp.br/mz/
}

GONÇALVES, R.B. \& BRANDÃO, C.R.F. Diversity of bees (Hymenoptera, Apidae) along a latitudinal gradient in the Atlantic Forest. Biota Neotrop. 8(4): http://www.biotaneotropica.org.br/v8n4/en/ abstract?article+bn00908042008

\begin{abstract}
The Atlantic Forest is one of the most rich and endangered natural environments in the world. It is expected then that this situation would have encouraged the study and conservation of this biome; the Atlantic forest Hymenoptera fauna, however, remains virtually unknown. The bee fauna, for instance, is less studied than that of the Brazilian open areas. The overall ignorance on most of the Atlantic Forest insect faunas based the elaboration of the project "Biodiversity of Hymenoptera and Isoptera: richness and diversity along a latitudinal gradient in the Atlantic Forest - the humid eastern forest of Brazil", within the Biota-Fapesp Program, that aimed to catalog the termites, ants and several wasp families along the Atlantic forest and to publish data that could contribute to the biome conservation. Similar sampling protocols were applied for wasp surveys: the same number of Malaise and pan traps and similar effort on vegetation swapping, along 17 selected localities, representing a gradient of almost $20^{\circ}$ of latitude in evergreen pristine Atlantic Forest, from Paraíba to Santa Catarina Brazilian states. These protocols were defined to optimize the collecting of wasps; bees represented a 'secondary product' of the sampling protocol. A sum of 797 specimens representing 105 bee species was sampled; the richest and more abundant bee taxon was the Meliponina. Unfortunately the applied sampling method does not afford comparisons among Atlantic Forest bee assemblages with those of other biomes. The detrended correspondence analysis using only the relative frequencies of Meliponina species revealed a weak correspondence of the stingless bee assemblages' composition with the latitudinal gradient. Despite that, the sampled taxa are possibly restricted to the Atlantic forest and additional data on their distribution deserves to be published. A complete checklist of the sampled bees, their relative frequencies, and the localities where they were recorded is presented and discussed.
\end{abstract}

Keywords: Apoidea, bee assemblages, Neotropical, surveys.

GONÇALVES, R.B. \& BRANDÃO, C.R.F. Diversidade de abelhas (Hymenoptera, Apidae) ao longo de um gradiente latitudinal na Mata Atlântica. Biota Neotrop. 8(4): http://www.biotaneotropica.org.br/v8n4/pt/ abstract?article+bn00908042008

Resumo: A Mata Atlântica é um dos ambientes mais ricos e ameaçados do mundo, o que deveria ter estimulado em muito o estudo e a conservação do Bioma, mas a fauna de Hymenoptera permanece ainda relativamente pouco conhecida. Em especial, a fauna de abelhas da floresta ombrófila densa é pouco estudada em comparação à fauna das áreas abertas brasileiras. O projeto temático "Biodiversidade de Hymenoptera e Isoptera: riqueza e diversidade ao longo de um gradiente latitudinal na Mata Atlântica - a floresta úmida do leste do Brasil”, integrante do Programa Biota-Fapesp, foi idealizado com o objetivo de catalogar térmitas, formigas e famílias selecionadas de vespas ao longo da Mata Atlântica, disponibilizando dados que permitam melhor embasar a conservação deste bioma. $\mathrm{O}$ protocolo de amostragem aplicada para a coleta de himenópteros (excluindo as formigas) empregou armadilhas Malaise, pratos-armadilha de cor amarela e esforço similar na varredura de vegetação ao longo de 17 localidades selecionadas, representando um gradiente de quase $20^{\circ}$ de latitude na Mata Atlântica, dos Estados da Paraíba até Santa Catarina. Este protocolo foi definido para otimizar a coleta de vespas, sendo as abelhas um produto secundário da amostragem (levantamentos de abelhas em geral utilizam captura em flores ao longo das estações do ano). No entanto, devido à escala regional do projeto e ao grande esforço de amostragem, uma expressiva quantidade de abelhas foi coletada durante o projeto, incluindo novos registros de abelhas para a Mata Atlântica. Foi amostrado um total de 797 espécimes distribuídos em 105 espécies de abelhas; o grupo de abelhas mais rico e abundante foi Meliponina. Uma análise de correspondência "destendenciada" ('detrended correspondence analysis') aplicada à uma matriz de presença ou ausência de Meliponina revela a relativamente fraca influência do gradiente latitudinal na composição das assembléias de abelhas de Mata Atlântica. Uma listagem das espécies amostradas por localidade, com suas freqüências relativas, é apresentada e discutida.

Palavras-chave: Apoidea, assembléias de abelhas, levantamentos, Neotrópico. 


\section{Introdução}

A Mata Atlântica brasileira é ao mesmo tempo um dos biomas mais biodiversos do mundo e um dos mais ameaçados pela ação antrópica (Myers et al. 2000), sendo um dos 34 hotspots mundiais e área prioritária para a conservação (Conservation International do Brasil et al. 2000). Como exemplos da diversidade da Mata Atlântica, estima-se que nela ocorra metade das espécies de mamíferos brasileiros (Fonseca et al. 1996); que abrigue uma avifauna comparativamente muito rica com 1020 espécies registradas, sendo 200 endêmicas (Agnello 2007) e, estudos recentes avaliam que o número de bactérias nas folhas possa ser de três a seis vezes o número estimado de espécies para oceanos e solo (Lambais et al. 2006). Para as abelhas, o número de espécies coletadas em uma única localidade da Mata Atlântica paulista (Wilms 1995), na Estação Biológica de Boracéia, em Salesópolis, é superior ao número de espécies coletadas em qualquer outra localidade na região Neotropical, incluindo áreas secas, onde, coletivamente, a riqueza de abelhas é considerada comparativamente superior (Michener 1979).

Quanto à ameaça humana, cabe notar que a área coberta pela Mata Atlântica era de aproximadamente $1.300 .000 \mathrm{~km}^{2}$ antes da colonização européia, e após mais de 500 anos de ocupação, a área preservada é estimada em cerca de $98.000 \mathrm{~km}^{2}$ (Morellato \& Haddad 2000), muito fragmentada, em especial na sua porção norte. Felizmente, dados recentes apontam para uma diminuição do ritmo de desmatamento em determinadas regiões com remanescentes de floresta (Conservation International do Brasil et al. 2000).

Tais aspectos deveriam ter estimulado pesquisas envolvendo a biota da Mata Atlântica, no entanto, a sua fauna de insetos, particularmente Hymenoptera e Isoptera, é pouco conhecida em relação à dos demais biomas brasileiros, como o Cerrado, por exemplo. Os fragmentos remanescentes de Mata Atlântica cobrem um gradiente latitudinal natural de quase 20 graus em um eixo norte-sul, da $\mathrm{Pa}$ raíba a Santa Catarina. Na verdade, esta situação representa uma oportunidade de enriquecer o acervo testemunho destes insetos ao longo do gradiente, a partir de inventários estruturados (Longino \& Colwell 1997) que permitem análises comparativas e identificações de padrões biogegráficos, ao mesmo tempo, aumentando o conhecimento taxonômico sobre estes insetos. O projeto temático "Biodiversidade de Hymenoptera e Isoptera: riqueza e diversidade ao longo de um gradiente latitudinal na Mata Atlântica - a floresta úmida do leste do Brasil", integrante do Programa Biota-Fapesp, pretendeu responder como as diferentes síndromes biológicas adotadas pelas diferentes famílias de Hymenoptera e Isoptera respondem ao gradiente latitudinal na Mata Atlântica e verificar se a partição biogeográfica do bioma, indicada por estudos de outros organismos, é confirmada pelas distribuições dos táxons selecionados para estudo pelo projeto. A principal ferramenta para estas análises é o conjunto de dados resultante de levantamentos qualitativos e/ou quantitativos de térmitas e famílias selecionadas de himenópteros ao longo de localidades de Mata Atlântica; além das análises, o projeto pretende disponibilizar dados que podem ser decisivos para fundamentar programas objetivando a conservação deste bioma.

Alguns grupos de organismos como, por exemplo, as aves (Gaston 2000), apresentam tendência de serem mais ricos nas porções tropicais do globo do que em áreas subtropicais e temperadas (ex. Brown \& Lomolino 1998). Este padrão de gradiente é conhecido como associação negativa entre riqueza de espécies e latitude ou padrão clássico de gradiente latitudinal, e é corroborado padrões exibidos por muitos táxons (Willig et al. 2003). Para plantas, Oliveira-Filho $\&$ Fontes (2000) reconhecem uma diferenciação norte-sul da flora arbórea na Mata Atlântica, possivelmente relacionada com a temperatura e o regime de chuvas. Exceções a este padrão clássico são também conhecidos para muitos organismos (ver Willig et al. 2003), por exemplo, Ichneumonidae que apresenta associação positiva entre riqueza e latitude (ex. Janzen 1981). Os Apidae de uma maneira geral apresentam tendência oposta - as porções temperadas e xéricas do mundo parecem ser mais ricas que as porções tropicais (Michener 1979). De qualquer maneira é esperado que a maioria dos organismos apresente certa variação em sua distribuição de riqueza segundo um gradiente latitudinal. Porém os padrões de distribuição revelados, em geral não se adequam perfeitamente ao gradiente devido às influências de outras variáveis geográficas (Gaston 2000), sendo chamados portanto de disruptivos.

Dados preliminares resultantes do inventariamento dos Formicidae amostrados pelo Projeto (neste caso em 26 localidades) apontam para uma diferenciação latitudinal da fauna e uma separação entre a composição do Nordeste e do Sudeste/Sul da Mata Atlântica (Silva et al. 2007). Os resultados preliminares do inventariamento de Isoptera, em 15 localidades, verificam a existência de um padrão clássico de gradiente latitudinal, com a separação das composições do Nordeste mais do estado do Espírito Santo daquelas do Sudeste/ Sul da Mata Atlântica (Cancello 2006, Cancello et al. 2006). Sob abordagem da biogeografia cladística, a diferenciação regional das áreas da Floresta sugere uma derivação histórica do norte para o sul da Mata Atlântica (Amorim \& Pires 1996, Costa et al. 2000, Pinto da Rocha et al. 2005, Silva et al. 2004).

Dentre os estudos de ecologia de comunidades de abelhas, inventários padronizados são relativamente comuns; entre 1970 quando se iniciaram e 2002, foram catalogados mais de 60 trabalhos no Brasil (Pinheiro-Machado 2002). Porém são poucos os inventários de abelhas da Mata Atlântica em comparação aos estudos em áreas de Cerrado, Caatinga e Campos Sulinos. Essa relativa escassez de levantamentos em áreas cobertas por floresta deve-se em grande parte à dificuldade da captura de abelhas em flores no dossel. Os levantamentos existentes na Mata Atlântica concentram-se na porção sul, em restingas continentais e insulares dos Estados do Paraná (Barbola 2000, Laroca 1974, Schwartz-Filho \& Laroca 1998), Santa Catarina (Steiner et al. 2006) e em florestas de São Paulo (Ramalho 1995, Wilms 1995). A estratégia adotada pelo presente projeto temático levou em conta a obtenção de um número representativo de amostras, obtidas pela aplicação de técnicas de coleta quantitativas em um número representativo de localidades ao longo do gradiente. Levantamentos de abelhas, entretanto, requerem a permanência em uma área determinada por períodos relativamente longos, pois exigem captura ativa e individual de visitantes florais após a localização e acesso a floradas, devendo levar em conta a sazonalidade (Sakagami et al. 1967).

O objetivo do presente trabalho é apresentar a listagem das abelhas coletadas ao longo do gradiente latitudinal através do projeto supracitado. Pelas razões explicadas acima, discutimos grande parte os resultados de forma qualitativa; apenas para espécies de abelhas sem ferrão foi possível uma análise quantitativa, por ser um grupo abundante e perene.

\section{Material e Métodos}

A Tabela 1 lista as 17 localidades sujeitas a amostragens de vespas pelo Projeto temático, segundo seu município e Estado e, quando for o caso, as respectivas unidades de conservação em que os locais de coleta estão inseridos, e os meses nos quais foram realizadas as amostragens. Os períodos de coleta deram-se preferencialmente nos meses chuvosos, que em geral mostram maior riqueza faunística; portanto, diferentes regiões do gradiente podem ter sido amostrados em diferentes períodos. O protocolo de coleta de vespas prevê a aplicação de um número fixo de armadilhas Malaise e pratos-armadilha (armadilha 
Abelhas ao longo de um gradiente latitudinal na Mata Atlântica

Tabela 1. Localidades amostradas ao longo de um gradiente latitudinal da Mata Atlântica do norte para o sul e respectivos meses de amostragem.

Table 1. Sampled localities along a north-south latitudinal gradient in the Atlantic Forest and respective sampling months.

\begin{tabular}{|c|c|c|c|c|}
\hline $\mathbf{N}$ & Coordenadas geográficas & Município - Estado & Localidade & Mês de amostragem \\
\hline L1 & $07^{\circ} 08^{\prime} 25^{\prime \prime} \mathrm{S}$ e $34^{\circ} 51^{\prime} 38^{\prime \prime} \mathrm{W}$ & João Pessoa - PB & Mata do Buraquinho & Julho de 2002 \\
\hline L2 & $08^{\circ} 00^{\prime} 33.9^{\prime \prime} \mathrm{S}$ e $34^{\circ} 56^{\prime} 31.5^{\prime \prime} \mathrm{W}$ & Recife - PE & Parque dos Dois Irmãos & Agosto de 2002 \\
\hline L3 & $09^{\circ} 19^{\prime} \mathrm{S}$ e $36^{\circ} 28^{\prime} \mathrm{W}$ & Quebrangulo - AL & Reserva Biológica Pedra Talhada & Setembro de 2002 \\
\hline L4 & $11^{\circ} 22^{\prime} 43.9^{\prime \prime} \mathrm{S}$ e $37^{\circ} 25^{\prime} 03.0^{\prime \prime} \mathrm{W}$ & Santa Luzia do Itanhy - SE & Crasto & Julho-Agosto de 2001 \\
\hline L5 & $12^{\circ} 33^{\prime} 40^{\prime \prime} \mathrm{S}$ e $38^{\circ} 02^{\prime} 44.8^{\prime \prime} \mathrm{W}$ & Mata de São João - BA & Reserva de Sapiranga & Julho de 2001 \\
\hline L6 & $14^{\circ} 46^{\prime} \mathrm{S}$ e $39^{\circ} 04^{\prime \prime} \mathrm{W}$ & Ilhéus - BA & Mata Esperança & Maio de 2002 \\
\hline L7 & $16^{\circ} 23^{\prime} 17.6^{\prime \prime} \mathrm{S}$ e $39^{\circ} 10^{\prime} 55.6^{\prime \prime} \mathrm{W}$ & Porto Seguro - BA & Estação Ecológica Pau Brasil & Maio de 2002 \\
\hline L8 & $18^{\circ} 58^{\prime} 03.0^{\prime \prime} \mathrm{S}$ e $40^{\circ} 08^{\prime} 03.6^{\prime \prime} \mathrm{W}$ & Linhares - ES & Reserva Biológica de Sooretama & Março de 2002 \\
\hline L9 & $19^{\circ} 58^{\prime} 18.5^{\prime \prime} \mathrm{S}$ e $40^{\circ} 32^{\prime} 07.6^{\prime \prime} \mathrm{W}$ & Santa Teresa - ES & Estação Biológica Santa Lúcia & Abril de 2001 \\
\hline L10 & $21^{\circ} 59^{\prime} 03.9^{\prime \prime} \mathrm{S}$ e $41^{\circ} 57^{\prime} 08.4^{\prime \prime} \mathrm{W}$ & Santa Maria Madalena - RJ & Parque Estadual do Desengano & Abril de 2002 \\
\hline L11 & $22^{\circ} 34^{\prime} 35^{\prime \prime} \mathrm{S}$ e $43^{\circ} 26^{\prime} 10^{\prime \prime} \mathrm{W}$ & Nova Iguaçu - RJ & Reserva Biológica do Tinguá & Março de 2002 \\
\hline L12 & $23^{\circ} 21^{\prime} 43^{\prime \prime} \mathrm{S}$ e $44^{\circ} 49^{\prime} 22^{\prime \prime} \mathrm{W}$ & Ubatuba-SP & $\begin{array}{l}\text { Parque Estadual da Serra do Mar, } \\
\text { Núcleo Picinguaba }\end{array}$ & Janeiro de 2002 \\
\hline L13 & $23^{\circ} 39^{\prime} 08.3^{\prime \prime} \mathrm{S}$ e $45^{\circ} 53^{\prime} 48.9^{\prime \prime} \mathrm{W}$ & Salesópolis - SP & Estação Ecológica Boracéia & Março-Abril de 2001 \\
\hline L14 & $24^{\circ} 18^{\prime} 16^{\prime \prime} \mathrm{S}$ e $48^{\circ} 21^{\prime} 53^{\prime \prime} \mathrm{W}$ & Ribeirão Grande - SP & $\begin{array}{l}\text { Parque Estadual Intervales, } \\
\text { Base Barra Grande }\end{array}$ & Dezembro de 2000 \\
\hline L15 & $24^{\circ} 31^{\prime} 06^{\prime \prime} \mathrm{S}$ e $47^{\circ} 12^{\prime} 06^{\prime \prime} \mathrm{W}$ & Peruíbe - SP & Estação Ecológica Juréia - Itatins & Abril-Maio de 2002 \\
\hline L16 & $25^{\circ} 34^{\prime} 37.2^{\prime \prime}$ e $48^{\circ} 53^{\prime} 53.7^{\prime \prime} \mathrm{W}$ & Morretes - PR & Parque Estadual do Pau Oco & Abril de 2002 \\
\hline L17 & $26^{\circ} 19^{\prime} 25^{\prime \prime} \mathrm{S}$ e $49^{\circ} 18^{\prime} 26.5^{\prime \prime} \mathrm{W}$ & São Bento do Sul - SC & CEPA - Rugendas & Outubro de 2001 \\
\hline
\end{tabular}

de Moericke) em cada localidade, assim como a aplicação do mesmo esforço na varredura da vegetação nestas localidades. Esta metodologia foi escolhida para privilegiar a coleta de vespas, sendo que as abelhas resultam em um produto secundário da amostragem.

Em cada área foram instaladas 10 armadilhas Malaise distribuídas em duas fileiras, uma em trilha pré-existente e outra dentro da floresta. Os pratos-armadilha de cor amarela foram distribuídos em grupos de 10 a cada lado de cinco pontos da trilha, somando 100 armadilhas em cada localidade. Os grupos de armadilhas foram separados por aproximadamente 100 metros entre si e as armadilhas mantidas disponíveis por um período de três dias. As coletas ativas não seletivas foram realizadas através da varredura da vegetação, utilizando redes entomológicas nas proximidades dos locais onde foram instaladas as armadilhas, mantendo o mesmo esforço empregado a cada localidade, padronizando o tempo gasto na atividade. As abelhas coletadas foram montadas e rotuladas com etiquetas de procedência e de numeração sequenciada, para fins de planilhamento, relacionando os dados individuais. Em seguida, foram separadas em morfo-espécies e comparadas com material identificado por especialistas, depositado no Museu de Zoologia da Universidade de São Paulo, São Paulo (MZSP). Alguns grupos de abelhas foram enviados a especialistas para identificação (vide Agradecimentos). O material está depositado na coleção do MZSP, exceto alguns exemplares de Meliponina em duplicata, depositados na coleção do Departamento de Biologia, Faculdade de Filosofia, Ciências e Letras de Ribeirão Preto, Universidade de São Paulo, Coleção João Maria Franco de Camargo, Ribeirão Preto.

A partir dos dados originais foi construída uma matriz de presença/ausência de Meliponina para cada localidade. Neste caso, o Parque Estadual de Intervales (Ribeirão Grande, SP) foi excluído já que nenhum Meliponina foi ali coletado. Esta matriz foi submetida à uma análise de correspondência "destendenciada" (detrended correspondence analysis, DCA) para avaliar as relações de similaridade entre as localidades ao longo do gradiente; a DCA é comumente utilizada para representar padrões de substituição gradual de espécies ao longo de gradientes ambientais (Ter Braak 1995). Diferentemente de outras técnicas de ordenação, a análise de correspondência ordena simultaneamente amostras e táxons; táxons e amostras fortemente associados estarão representados por pontos próximos no plano da ordenação. A análise foi realizada pelo programa Vegan versão 1.8-8 (Oksanen et al. 2007).

\section{Resultados}

Em números absolutos foram coletados 797 espécimes de abelhas, dos quais 114 machos. Estes indivíduos estão distribuídos em 105 espécies, de 50 gêneros e 15 tribos, sendo que 50 espécies puderam ser nomeadas. A lista completa das espécies está na Tabela 2. Todas as subfamílias de Apidae presentes no Brasil foram amostradas, sendo que Apinae foi a mais rica com 54 espécies, das quais 30 correspondem a abelhas sem ferrão (Meliponina). As demais subfamílias, em ordem decrescente de riqueza foram Halictinae (38 espécies), Andreninae (cinco espécies), Megachilinae e Colletinae (quatro espécies cada). Em ordem de abundância decrescente estão os Apinae com 664 indivíduos, Halictinae com 91, Andreninae com 31, Colletinae com seis e Megachilinae com cinco indivíduos. Novamente, as abelhas sem ferrão merecem destaque por apresentarem uma elevada abundância relativa com 582 exemplares coletados.

As espécies mais abundantes foram Trigona aff. fulviventris com 202 indivíduos, seguida de Partamona criptica com 145 indivíduos, e Plebeia phrynostoma com 65 indivíduos (todos de Meliponina); os gêneros representados por estas espécies foram também os mais abundantes nesta ordem. Os gêneros mais ricos em espécies foram Dialictus (Halictinae, 10), seguido dos apíneos Plebeia (sete), Ceratina (seis), Osiris (cinco) e Melipona (quatro). Do total de espécies coletadas, 14\% são cleptoparasitas (15), distribuídas em cinco tribos. Gêneros de abelhas considerados comuns e com ampla distribuição e alta abundância, como Centris, Exomalopsis e Xylocopa e suas várias espécies de ocorrência conhecida na Mata Atlântica não foram registrados no presente trabalho. 
Gonçalves, R.B. \& Brandão, C.R.F.

Tabela 2. Listagem das espécies de abelhas coletadas ao longo de um gradiente latitudinal na Mata Atlântica (ver Material e Métodos).

Table 2. Checklist of the bees sampled along a latitudinal gradient in the Atlantic Forest (see Material e Métodos).

\begin{tabular}{|c|c|c|c|c|}
\hline $\mathbf{N}$ & Espécie & Localidade & Método de captura & Indivíduos \\
\hline \multicolumn{5}{|c|}{ ANDRENINAE } \\
\hline \multicolumn{5}{|c|}{ Protandrenini } \\
\hline 1 & Anthrenoides sp.1 & Salesópolis - SP & malaise & 1 fêmea \\
\hline 2 & Anthrenoides zanellai Urban, 2005 & São Bento do Sul - SC & malaise & 1 fêmea \\
\hline 3 & Rhophitulus sp.01 & Santa Maria Madalena - RJ & varredura & 5 fêmeas \\
\hline \multirow[t]{2}{*}{4} & Rhophitulus sp.02 & Quebrangulo - AL & malaise & 2 fêmeas e 1 macho \\
\hline & & & varredura & 4 fêmeas e 13 machos \\
\hline 5 & Rhophitulus sp.03 & Quebrangulo - AL & varredura & 4 machos \\
\hline \multicolumn{5}{|c|}{ APINAE } \\
\hline & Apini & & & \\
\hline \multirow[t]{11}{*}{6} & Apis mellifera Linnaeus, 1758 & Ilhéus - BA & pratos & 1 fêmea \\
\hline & & João Pessoa - PB & malaise & 1 fêmea \\
\hline & & Mata de São João - BA & malaise & 1 fêmea \\
\hline & & Morretes - PR & malaise & 1 fêmea \\
\hline & & Nova Iguaçu - RJ & malaise & 1 fêmea \\
\hline & & Porto Seguro - BA & malaise & 2 fêmeas \\
\hline & & Quebrangulo - AL & varredura & 3 fêmeas \\
\hline & & & malaise & 1 fêmea \\
\hline & & Santa Luzia do Itahy- SE & varredura & 1 fêmea \\
\hline & & São Bento do Sul - SC & malaise & 2 fêmeas \\
\hline & & Ubatuba - SP & malaise & 2 fêmeas \\
\hline 7 & Bombus (Fervidobombus) brasiliensis Lepeletier, 1836 & Ubatuba - SP & malaise & 1 fêmea \\
\hline \multirow[t]{2}{*}{8} & Bombus (Fervidobombus) morio (Swederus, 1787) & Ribeirão Grande - SP & malaise & 1 fêmea \\
\hline & & Ubatuba - SP & malaise & 2 fêmeas \\
\hline 9 & Euglossa (Glossurella) stellfeldi Moure, 1947 & Santa Teresa - ES & malaise & 1 fêmea \\
\hline 10 & Frieseomelitta doederleini (Friese, 1900) & Recife - PE & malaise & 1 fêmea \\
\hline 11 & Frieseomelitta dispar (Moure, 1950) & Mata de São João - BA & malaise & 2 fêmeas \\
\hline \multirow[t]{2}{*}{12} & Frieseomelitta francoi (Moure, 1946) & Mata de São João - BA & malaise & 1 fêmea \\
\hline & & Santa Luzia do Itahy- SE & malaise & 2 fêmeas \\
\hline \multirow[t]{3}{*}{13} & Lestrimelitta ehrhardti (Friese, 1931) & Nova Iguaçu - RJ & malaise & 1 macho \\
\hline & & Quebrangulo - AL & malaise & 2 machos \\
\hline & & Ubatuba - SP & malaise & 1 fêmea \\
\hline 14 & Lestrimelitta rufipes (Friese, 1903) & Santa Maria Madalena - RJ & malaise & 3 fêmeas \\
\hline 15 & Lestrimelitta tropica Marchi \& Melo, 2006 & Nova Iguaçu - RJ & malaise & 2 fêmeas \\
\hline \multirow[t]{2}{*}{16} & Leurotrigona muelleri (Friese, 1900) & Quebrangulo - AL & varredura & 1 fêmea \\
\hline & & Santa Maria Madalena - RJ & malaise & 1 fêmea \\
\hline \multirow[t]{2}{*}{17} & Melipona (Eomelipona) bicolor Lepeletier, 1836 & Santa Teresa - ES & malaise & 20 fêmeas \\
\hline & & São Bento do Sul - SC & malaise & 1 fêmea \\
\hline \multirow[t]{6}{*}{18} & Melipona (Eomelipona) marginata carioca Moure, 1971 & Nova Iguaçu - RJ & malaise & 2 fêmeas \\
\hline & & Quebrangulo - AL & malaise & 1 macho \\
\hline & & Salesópolis - SP & malaise & 1 fêmea \\
\hline & & Santa Teresa - ES & malaise & 2 fêmeas \\
\hline & & Ubatuba - SP & malaise & 2 fêmeas \\
\hline & & Linhares - ES & malaise & 1 fêmea \\
\hline \multirow[t]{3}{*}{19} & Melipona (Michmelia) mondury Smith, 1863 & Nova Iguaçu - RJ & malaise & 1 fêmea \\
\hline & & Porto Seguro - BA & malaise & 1 fêmea \\
\hline & & Ubatuba - SP & malaise & 2 fêmeas \\
\hline 20 & Melipona (Michmelia) scutellaris Latreille, 1811 & Santa Luzia do Itahy- SE & malaise & 1 fêmea \\
\hline 21 & Oxytrigona tataira (Smith, 1863) & Nova Iguaçu - RJ & malaise & 1 fêmea \\
\hline 22 & Paratrigona glabrata Moure, 1989 & Linhares - ES & malaise & 1 fêmea \\
\hline
\end{tabular}


Tabela 2. Continuação...

\begin{tabular}{|c|c|c|c|c|}
\hline $\mathbf{N}$ & Espécie & Localidade & Método de captura & Indivíduos \\
\hline & & Ilhéus - BA & malaise & 1 fêmea \\
\hline & & & varredura & 1 fêmea \\
\hline \multirow[t]{3}{*}{23} & Paratrigona subnuda Moure, 1947 & Nova Iguaçu - RJ & malaise & 2 fêmea \\
\hline & & Salesópolis - SP & malaise & 1 fêmea \\
\hline & & Santa Maria Madalena - RJ & varredura & 1 fêmea \\
\hline \multirow[t]{3}{*}{24} & Partamona criptica Pedro \& Camargo, 2003 & Ilhéus - BA & malaise & 86 fêmeas e 1 macho \\
\hline & & Porto Seguro - BA & malaise & 4 fêmea \\
\hline & & Santa Teresa - ES & malaise & 47 fêmeas e 7 machos \\
\hline \multirow[t]{8}{*}{25} & Partamona helleri (Friese, 1900) & João Pessoa - PB & malaise & 8 fêmeas \\
\hline & & Linhares - ES & malaise & 2 fêmeas \\
\hline & & Morretes - PR & malaise & 3 fêmeas \\
\hline & & Nova Iguaçu - RJ & malaise & 1 fêmea \\
\hline & & Recife - PE & malaise & 11 fêmeas \\
\hline & & Santa Luzia do Itahy- SE & malaise & 3 fêmeas \\
\hline & & Santa Maria Madalena - RJ & malaise & 2 fêmeas \\
\hline & & Ubatuba - SP & malaise & 1 fêmea \\
\hline \multirow[t]{3}{*}{26} & Plebeia lucii Moure, 2004 & João Pessoa - PB & malaise & 1 fêmea \\
\hline & & Linhares - ES & malaise & 3 fêmeas \\
\hline & & Santa Maria Madalena - RJ & varredura & 2 fêmeas \\
\hline \multirow[t]{3}{*}{27} & Plebeia poecilochroa Camargo \& Moure, 1989 & Ilhéus - BA & malaise & 3 fêmeas \\
\hline & & Linhares - ES & malaise & 1 fêmea \\
\hline & & Porto Seguro - BA & malaise & 2 fêmeas \\
\hline 28 & $\begin{array}{l}\text { Plebeia sp.01 } \\
\text { (aff. poecilochroa Camargo \& Moure, 1989) }\end{array}$ & Santa Luzia do Itahy- SE & malaise & 1 fêmea \\
\hline 29 & $\begin{array}{l}\text { Plebeia sp.02 } \\
\text { (aff. poecilochroa Camargo \& Moure, 1989) }\end{array}$ & Santa Maria Madalena - RJ & malaise & 1 fêmea \\
\hline \multirow[t]{14}{*}{30} & Plebeia phrynostoma Moure, 2004 & João Pessoa - PB & malaise & 11 fêmeas \\
\hline & & Mata de São João - BA & malaise & 1 fêmea \\
\hline & & Nova Iguaçu - RJ & malaise & 5 fêmeas \\
\hline & & Peruíbe - SP & varredura & 2 fêmeas \\
\hline & & Quebrangulo - AL & malaise & 1 fêmea \\
\hline & & & varredura & 28 fêmeas \\
\hline & & Recife - PE & malaise & 4 fêmeas \\
\hline & & Santa Luzia do Itahy- SE & malaise & 1 fêmea \\
\hline & & Santa Maria Madalena - RJ & malaise & 1 fêmea \\
\hline & & Santa Teresa - ES & malaise & 1 fêmea \\
\hline & & & pratos & 1 fêmea \\
\hline & & & varredura & 2 fêmeas \\
\hline & & Ubatuba - SP & malaise & 6 fêmeas \\
\hline & & & varredura & 1 fêmea \\
\hline \multirow[t]{6}{*}{31} & Plebeia remota (Holmberg, 1903) & Ubatuba - SP & malaise & 1 fêmea e 1 macho \\
\hline & & Morretes - PR & malaise & 4 fêmeas \\
\hline & & Nova Iguaçu - RJ & malaise & 3 fêmeas \\
\hline & & Peruíbe - SP & varredura & 2 fêmeas \\
\hline & & Santa Teresa - ES & malaise & 1 fêmea \\
\hline & & São Bento do Sul - SC & malaise & 1 fêmea \\
\hline 32 & Plebeia saiqui (Friese, 1900) & São Bento do Sul - SC & malaise & 1 fêmea \\
\hline 33 & Scaptotrigona sp.01 & Santa Luzia do Itahy- SE & malaise & 1 fêmea e 1 macho \\
\hline 34 & Scaptotrigona sp.02 & Nova Iguaçu - RJ & malaise & 1 macho \\
\hline 35 & Schwarziana quadripunctata (Lepeletier, 1836) & Nova Iguaçu - RJ & malaise & 1 fêmea \\
\hline 36 & Tetragonisca angustula (Latreille, 1811) & Ilhéus - BA & malaise & 1 fêmea \\
\hline
\end{tabular}


Tabela 2. Continuação...

\begin{tabular}{|c|c|c|c|c|}
\hline $\mathbf{N}$ & Espécie & Localidade & Método de captura & Indivíduos \\
\hline & & Nova Iguaçu - RJ & malaise & 4 fêmeas \\
\hline & & Quebrangulo - AL & varredura & 2 fêmeas \\
\hline & & Santa Luzia do Itahy- SE & malaise & 1 fêmea \\
\hline & & Santa Maria Madalena - RJ & varredura & 1 fêmea \\
\hline & & São Bento do Sul - SC & malaise & 1 fêmea \\
\hline \multirow[t]{16}{*}{37} & Trigona aff. fulviventris Guérin, 1835 & Ilhéus - BA & malaise & 84 fêmeas e 2 machos \\
\hline & & & varredura & 2 fêmeas \\
\hline & & João Pessoa - PB & malaise & 1 fêmea e 27 machos \\
\hline & & Nova Iguaçu - RJ & malaise & 4 fêmeas \\
\hline & & Peruíbe - SP & malaise & 3 fêmeas \\
\hline & & & varredura & 1 fêmea \\
\hline & & Porto Seguro - BA & varredura & 1 fêmea \\
\hline & & Quebrangulo - AL & varredura & 13 fêmeas \\
\hline & & & malaise & 13 fêmeas e 2 machos \\
\hline & & Recife - PE & malaise & 2 fêmeas \\
\hline & & Salesópolis - SP & malaise & 2 fêmeas \\
\hline & & Santa Luzia do Itahy- SE & malaise & 1 fêmea \\
\hline & & Santa Maria Madalena - RJ & malaise & 16 fêmeas e 1 macho \\
\hline & & Santa Teresa - ES & malaise & 23 fêmeas e 1 macho \\
\hline & & & pratos & 2 fêmeas \\
\hline & & Ubatuba - SP & malaise & 1 macho \\
\hline 38 & Trigona hyalinata (Lepeletier, 1836) & Linhares - ES & malaise & 1 fêmea \\
\hline \multirow[t]{15}{*}{39} & Trigona spinipes (Fabricius, 1793) & Ilhéus - BA & malaise & 2 fêmeas \\
\hline & & & varredura & 1 fêmea \\
\hline & & Linhares - ES & malaise & 1 fêmea \\
\hline & & Mata de São João - BA & pratos & 1 fêmea \\
\hline & & Morretes - PR & malaise & 1 fêmea \\
\hline & & & varredura & 1 fêmea \\
\hline & & Nova Iguaçu - RJ & malaise & 12 fêmeas \\
\hline & & & pratos & 1 fêmea \\
\hline & & Porto Seguro - BA & varredura & 1 fêmea \\
\hline & & Quebrangulo - AL & malaise & 1 fêmea \\
\hline & & & varredura & 2 fêmeas \\
\hline & & Santa Luzia do Itahy- SE & malaise & 7 fêmeas \\
\hline & & Santa Maria Madalena - RJ & malaise & 2 fêmeas \\
\hline & & Ubatuba - SP & malaise & 3 fêmeas \\
\hline & Eucerini & & & \\
\hline 40 & $\begin{array}{l}\text { Melissoptila richardiae Bertoni \& Schrottky, } 1910 \\
\text { Nomadini }\end{array}$ & Quebrangulo - AL & varredura & 1 fêmea \\
\hline \multirow[t]{2}{*}{41} & Brachynomada sp.01 & Quebrangulo - AL & malaise & 1 fêmea \\
\hline & & & varredura & 1 fêmea \\
\hline 42 & Pseudepeolus sp.01 & Quebrangulo - AL & varredura & 1 fêmea \\
\hline \multirow[t]{2}{*}{43} & Trophocleptria sp.01 & Santa Teresa - ES & malaise & 1 fêmea \\
\hline & Osirini & & & \\
\hline 44 & Osirinus sp.01 & Salesópolis - SP & malaise & 2 fêmeas \\
\hline \multirow[t]{4}{*}{45} & Osiris sp.01 & Linhares - ES & malaise & 2 machos \\
\hline & & Nova Iguaçu - RJ & malaise & 1 fêmea \\
\hline & & Santa Maria Madalena - RJ & malaise & 2 fêmeas \\
\hline & & Ubatuba - SP & malaise & 2 machos \\
\hline 46 & Osiris sp.02 & Peruíbe - SP & malaise & 1 fêmea \\
\hline
\end{tabular}


Tabela 2. Continuação...

\begin{tabular}{|c|c|c|c|c|}
\hline $\mathbf{N}$ & Espécie & Localidade & Método de captura & Indivíduos \\
\hline & & Santa Teresa - ES & malaise & 1 fêmea \\
\hline \multirow[t]{2}{*}{47} & Osiris sp.03 & Ribeirão Grande - SP & malaise & 3 fêmea \\
\hline & & Santa Maria Madalena - RJ & malaise & 2 fêmea \\
\hline 48 & Osiris sp.04 & Ribeirão Grande - SP & malaise & 1 fêmea \\
\hline \multirow[t]{2}{*}{49} & Osiris sp.05 & João Pessoa - PB & malaise & 1 fêmea \\
\hline & & Recife - PE & malaise & 1 fêmea \\
\hline 50 & Protosiris sp.01 & Santa Maria Madalena - RJ & malaise & 1 macho \\
\hline \multirow[t]{2}{*}{51} & Protosiris sp.02 & Nova Iguaçu - RJ & malaise & 3 fêmeas \\
\hline & Tapinotaspidini & & & \\
\hline \multirow[t]{4}{*}{52} & Paratetrapedia bicolor (Smith, 1854) & Ilhéus - BA & varredura & 1 fêmea \\
\hline & & Linhares - ES & varredura & 1 fêmea \\
\hline & & Quebrangulo - AL & varredura & 1 fêmea \\
\hline & & Santa Luzia do Itahy- SE & malaise & 1 fêmea \\
\hline \multirow[t]{2}{*}{53} & Lophopedia pygmaea (Schrottky, 1902) & Santa Teresa - ES & varredura & 1 fêmea \\
\hline & Xylocopini & & & \\
\hline \multirow[t]{6}{*}{54} & Ceratina (Ceratinula) sp.01 & Morretes - PR & malaise & 1 fêmea \\
\hline & & & varredura & 1 fêmea \\
\hline & & Nova Iguaçu - RJ & varredura & 1 fêmea e 2 machos \\
\hline & & Ribeirão Grande - SP & malaise & 2 fêmeas \\
\hline & & Santa Maria Madalena - RJ & varredura & 1 fêmea \\
\hline & & Ubatuba - SP & malaise & 7 fêmeas e 5 machos \\
\hline 55 & Ceratina (Ceratinula) sp.02 & João Pessoa - PB & malaise & 1 macho \\
\hline 56 & Ceratina (Ceratinula) sp.03 & Quebrangulo - AL & varredura & 2 fêmeas \\
\hline 57 & Ceratina (Crewella) sp.01 & Peruíbe - SP & malaise & 1 fêmea \\
\hline 58 & Ceratina (Crewella) sp.02 & Quebrangulo - AL & varredura & 2 fêmeas \\
\hline 59 & Ceratina (Crewella) sp.03 & Ilhéus - BA & malaise & 1 fêmea \\
\hline \multicolumn{5}{|c|}{ COLLETINAE } \\
\hline & Colletini & & & \\
\hline \multirow[t]{2}{*}{60} & Colletes rugicollis Friese, 1900 & Ubatuba - SP & malaise & 1 fêmea \\
\hline & Hylaeini & & & \\
\hline 61 & Hylaeus (Hylaeopsis) sp.01 & Santa Maria Madalena - RJ & malaise & 1 fêmea \\
\hline \multirow[t]{2}{*}{62} & Hylaeus (Hylaeopsis) sp.02 & Nova Iguaçu - RJ & malaise & 1 fêmea \\
\hline & Xeromelissini & & & \\
\hline \multirow[t]{2}{*}{63} & Chilicola (Hylaeosoma) megalostigma (Ducke, 1908) & Ilhéus - BA & malaise & 1 fêmea e 1 macho \\
\hline & & Quebrangulo - AL & varredura & 1 fêmea \\
\hline \multicolumn{5}{|c|}{ HALICTINAE } \\
\hline & Augochlorini & & & \\
\hline \multirow[t]{2}{*}{64} & Ariphanarthra palpalis Moure, 1951 & Morretes - PR & malaise & 2 machos \\
\hline & & Ribeirão Grande - SP & malaise & 3 fêmeas \\
\hline 65 & Augochlora (Augochlora) dolichocephala (Moure, 1941) & São Bento do Sul - SC & malaise & 2 fêmeas \\
\hline 66 & Augochlora (Augochlora) sp.01 & São Bento do Sul - SC & malaise & 1 fêmea \\
\hline 67 & Augochlora (Oxystoglossella) morrae Strand, 1900 & São Bento do Sul - SC & varredura & 1 fêmea \\
\hline 68 & Augochlorella ephyra (Schrottky, 1910) & Quebrangulo - AL & varredura & 1 fêmea \\
\hline \multirow[t]{3}{*}{69} & Augochlorella urania (Smith, 1853) & Salesópolis - SP & malaise & 1 macho \\
\hline & & São Bento do Sul - SC & malaise & 1 fêmea \\
\hline & & Ubatuba - SP & varredura & 1 macho \\
\hline 70 & Augochloropsis sp.01 & Mata de São João - BA & malaise & 6 fêmeas \\
\hline 71 & Augochloropsis sp.02 & Ilhéus - BA & malaise & 2 fêmeas \\
\hline 72 & Augochloropsis sp.03 & Quebrangulo - AL & varredura & 1 fêmea \\
\hline 73 & Megalopta sp.01 & Nova Iguaçu - RJ & varredura & 1 macho \\
\hline
\end{tabular}


Tabela 2. Continuação...

\begin{tabular}{|c|c|c|c|c|}
\hline $\mathbf{N}$ & Espécie & Localidade & Método de captura & Indivíduos \\
\hline 74 & Megalopta sp.02 & Santa Luzia do Itahy- SE & varredura & 1 macho \\
\hline 75 & Megalopta sp.03 & João Pessoa - PB & malaise & 1 fêmea \\
\hline 76 & Megaloptina festivaga (Dalla Torre, 1896) & João Pessoa - PB & malaise & 1 macho \\
\hline 77 & Megommation insigne (Smith, 1853) & São Bento do Sul - SC & pratos & 1 fêmea \\
\hline \multirow[t]{2}{*}{78} & Neocorynura aenigma (Gribodo, 1894) & Morretes - PR & malaise & 1 fêmea \\
\hline & & São Bento do Sul - SC & malaise & 1 fêmea e 3 machos \\
\hline 79 & Neocorynura sp.01 & Santa Maria Madalena - RJ & malaise & 1 fêmea \\
\hline 80 & Neocorynura sp.02 & Ubatuba - SP & malaise & 1 fêmea \\
\hline 81 & Pereirapis semiaurata (Spinola, 1853) & Ilhéus - BA & varredura & 6 machos \\
\hline 82 & Rhectomia sp.01 & Ubatuba - SP & malaise & 1 fêmea \\
\hline 83 & Rhectomia sp.02 & Quebrangulo - AL & varredura & 1 macho \\
\hline 84 & Temnosoma sp.01 & Mata de São João - BA & varredura & 1 fêmea \\
\hline 85 & Temnosoma sp.02 & Ubatuba - SP & malaise & 1 fêmea \\
\hline \multirow[t]{2}{*}{86} & Gênero e espécie não determinados* & Salesópolis - SP & malaise & 1 macho \\
\hline & Halictini & & & \\
\hline 87 & Caenohalictus oresicoetes (Moure, 1943) & Salesópolis - SP & malaise & 4 fêmea \\
\hline 88 & Dialictus sp.01 & Salesópolis - SP & malaise & 4 fêmea \\
\hline \multirow[t]{2}{*}{89} & Dialictus sp.02 & Salesópolis - SP & varredura & 1 fêmea \\
\hline & & Ubatuba - SP & malaise & 1 fêmea \\
\hline 90 & Dialictus sp.03 & Ilhéus - BA & varredura & 8 machos \\
\hline 91 & Dialictus sp.04 & Ilhéus - BA & varredura & 1 macho \\
\hline 92 & Dialictus sp.05 & Nova Iguaçu - RJ & malaise & 2 fêmeas \\
\hline 93 & Dialictus sp.06 & São Bento do Sul - SC & malaise & 5 fêmeas \\
\hline 94 & Dialictus sp.07 & Nova Iguaçu - RJ & malaise & 5 fêmeas \\
\hline 95 & Dialictus sp.08 & Nova Iguaçu - RJ & malaise & 2 fêmeas \\
\hline 96 & Dialictus sp.09 & Nova Iguaçu - RJ & malaise & 1 fêmea \\
\hline \multirow[t]{2}{*}{97} & Dialictus sp.10 & Morretes - PR & malaise & 1 fêmea \\
\hline & & Ribeirão Grande - SP & malaise & 1 fêmea \\
\hline \multirow[t]{3}{*}{98} & Habralictus canaliculatus Moure, 1941 & Nova Iguaçu - RJ & varredura & 1 macho \\
\hline & & Santa Maria Madalena - RJ & malaise & 2 fêmea \\
\hline & & Santa Teresa - ES & malaise & 1 fêmea \\
\hline 99 & Habralictus macrospilophorus Moure, 1941 & Santa Maria Madalena - RJ & malaise & 2 macho \\
\hline 100 & $\begin{array}{l}\text { Microsphecodes russeiclypeatus } \\
\text { (Sakagami \& Moure, 1952) }\end{array}$ & Peruíbe - SP & varredura & 1 fêmea \\
\hline 101 & Sphecodes sp.01 & Nova Iguaçu - RJ & malaise & 1 fêmea \\
\hline \multicolumn{5}{|c|}{ MEGACHILINAE } \\
\hline \multicolumn{5}{|c|}{ Anthidiini } \\
\hline 102 & Anthodioctes megachiloides Holmberg, 1903 & São Bento do Sul - SC & malaise & 1 fêmea \\
\hline \multirow[t]{2}{*}{103} & Moureanthidium capixaba Urban, 1995 & Santa Maria Madalena - RJ & varredura & 1 fêmea \\
\hline & Megachilini & & & \\
\hline 104 & Coelioxys (Cyrtocoelioxys) sp.01 & Ubatuba - SP & malaise & 2 fêmeas \\
\hline 105 & Megachile (Moureapis) sp.01 & Quebrangulo - AL & varredura & 1 fêmea \\
\hline
\end{tabular}

* Gênero e espécie não determinados, pertencente possivelmente ao grupo de gêneros relacionados com Megaloptidia.

* Undetermined genus and species, possibly belonging to genera related to Megaloptidia.

A técnica mais efetiva na captura de abelhas foi a armadilha Malaise, com 647 indivíduos de 84 espécies coletados, seguida da varredura da vegetação com 143 indivíduos de 39 espécies; os pratos-armadilha capturaram apenas sete indivíduos de cinco espécies (quatro corbiculadas mais uma fêmea do halictíneo Megommation insigne). Quanto às armadilhas Malaise, aquelas instaladas dentro das florestas (251 indivíduos capturados, 41 espécies) se mostraram menos eficientes que as instaladas nas trilhas (383 indivíduos, 58 espécies).

A Tabela 3 apresenta o número de indivíduos e espécies de abelhas coletadas em cada localidade, em geral e de Meliponina em particular. Os resultados da análise da DCA estão expressos na Figura 1. 
Tabela 3. Riqueza e abundância de abelhas ao longo de um gradiente latitudinal na Mata Atlântica. Número de espécies $(\mathrm{S})$ e indivíduos $(\mathrm{N})$ totais e apenas para Meliponina ( $\mathrm{S}_{\text {Meliponina, }} \mathrm{N}_{\text {meliponina }}$ ).

Table 3. Richness and abundance of bees along a latitudinal gradient in Atlantic Forest. Number of species (S) and individuals $(\mathrm{N})$ and Meliponina richness and abundance $\left(\mathrm{S}_{\text {Meliponina, }} \mathrm{N}_{\text {meliponina }}\right)$.

\begin{tabular}{llrrrc}
\hline N & \multicolumn{1}{c}{ Município/Estado } & S & $\mathbf{S}_{\text {Meliponina }}$ & N & $\mathbf{N}_{\text {Meliponina }}$ \\
\hline L1 & João Pessoa - PB & 9 & 4 & 53 & 48 \\
L2 & Recife - PE & 5 & 4 & 20 & 19 \\
L3 & Quebrangulo - AL & 22 & 7 & 115 & 66 \\
L4 & Santa Luzia do Itanhy - SE & 12 & 9 & 22 & 19 \\
L5 & Mata de São João - BA & 7 & 4 & 13 & 5 \\
L6 & Ilhéus - BA & 14 & 6 & 201 & 184 \\
L7 & Porto Seguro - BA & 6 & 5 & 11 & 9 \\
L8 & Linhares - ES & 9 & 7 & 13 & 10 \\
L9 & Santa Teresa - ES & 11 & 6 & 112 & 107 \\
L10 & Santa Maria Madalena - RJ & 20 & 10 & 49 & 31 \\
L11 & Nova Iguaçu - RJ & 27 & 14 & 64 & 41 \\
L12 & Ubatuba-SP & 21 & 8 & 46 & 18 \\
L13 & Salesópolis - SP & 10 & 3 & 17 & 4 \\
L14 & Ribeirão Grande - SP & 6 & 0 & 11 & 0 \\
L15 & Peruíbe - SP & 6 & 3 & 11 & 8 \\
L16 & Morretes - PR & 8 & 3 & 16 & 9 \\
L17 & São Bento do Sul - SC & 14 & 4 & 23 & 4 \\
& Total geral & 105 & 30 & 797 & 582 \\
\hline
\end{tabular}

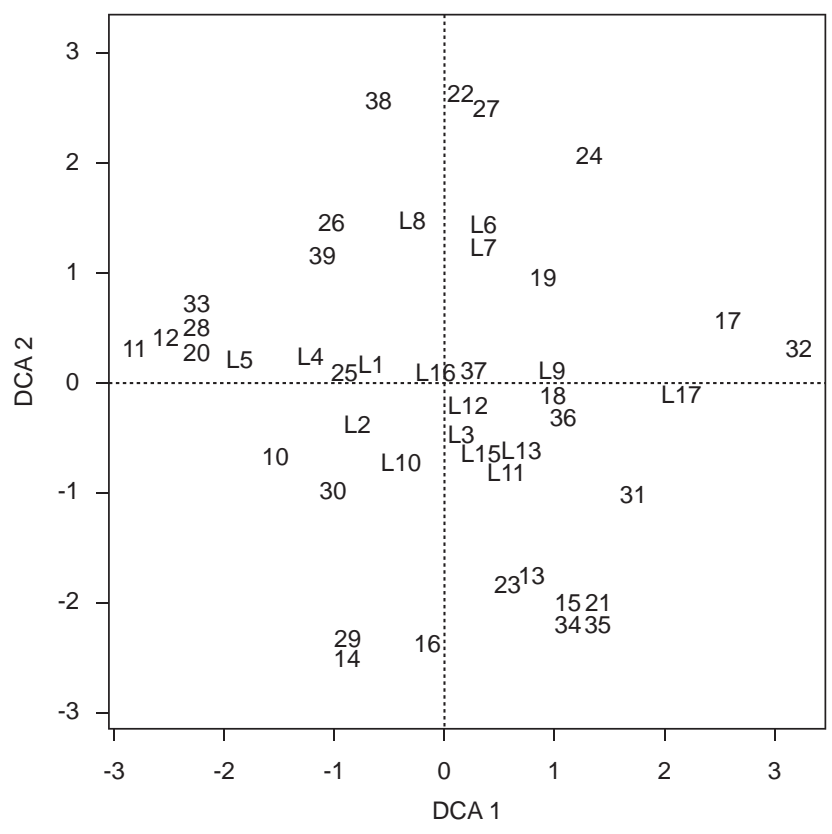

Figura 1. Dois primeiros eixos da análise de correspondência "destendenciada". Letra "L" seguida de números grandes em negrito correspondem às localidades amostradas (códigos conforme Tabela 1), números pequenos correspondem as espécies de Meliponina (códigos conforme Tabela 2).

Figure 1. Two first axes of detrended correspondence analysis. Large bold numbers preceded by "L" correspond to the sampled localities (codes as Table 1), small numbers correspond to the Meliponina species (codes as Table 2).

\section{Discussão}

O número de espécies coletadas apresentou grande variação em torno da média de 12 espécies em cada localidade. As localidades do Estado do Rio de Janeiro estiveram entre as mais ricas (com 20 ou mais espécies), assim como uma localidade em São Paulo (Ubatuba) e em Alagoas. De uma maneira geral, Meliponina apresentou uma riqueza relativamente elevada, especialmente nas áreas do Rio de Janeiro até Paraíba; em média, metade de todas as espécies coletadas em cada localidade foi de abelhas sem ferrão. As localidades amostradas em Porto Seguro (BA) e Recife (PE) são exemplos de predomínio de Meliponina (Tabela 3); as localidades em São Bento do Sul (SC) e Ribeirão Grande (SP) são exemplos opostos; na última delas nenhuma espécie de Meliponina foi coletada. Da mesma maneira, nota-se uma grande variação no número de indivíduos coletados entre as localidades, tanto para as abelhas como um todo como para Meliponina. Em torno de $62 \%$ dos indivíduos em uma dada localidade corresponde às abelhas sem ferrão. O número relativamente elevado de espécies e indivíduos coletados de Meliponina em comparação com outras abelhas deve ser relacionado a limitações das técnicas de amostragem utilizadas, que são mais eficientes para abelhas sem ferrão, visto que a maioria das suas espécies vive em colônias perenes e com elevada densidade, aumentando suas chances de serem coletadas.

A utilização de armadilhas Malaise pode ser uma alternativa para amostragem estatística de abelhas ou uma forma de complementar amostragens, especialmente em áreas florestadas devido à dificuldade de acesso a flores no dossel; o emprego de armadilhas permite ainda a bem-vinda captura de cleptoparasitas e Meliponina. É plausível que o emprego deste tipo de armadilha contribua com uma informação útil sobre a melissofauna de determinada localidade, mas seu emprego exclusivo possivelmente subestime a riqueza total, como já foi constatado para Ichneumonidae (Fraser et al. 2008). Armadilhas não são seletivas e capturam muitas ordens e famílias de insetos, sendo seu emprego adequado em inventários que objetivem diversificar os táxons a serem amostrados. Já o emprego de pratos-armadilha teve resultado insatisfatório em relação à riqueza esperada. Dentre os levantamentos feitos no Brasil, apenas recentemente os pratos-armadilha foram empregados em inventariamentos (Krug \& Alves-dos-Santos 2008, Souza \& Campos 2008). Krug \& Alves-dos-Santos (2008) relatam uma eficiência relativamente elevada dos pratos-armadilha em comparação à captura em flores; nesta linha, Roulston et al. (2007) sugerem o emprego desta técnica quando as flores são escassas ou quando é desejada a amostragem de espécies particulares de abelhas que são atraídas pelos pratos-armadilhas.

A análise de correspondência "destendenciada" (DCA) revelou um padrão que expressa, em parte, um efeito do gradiente latitudinal na composição de espécies (Figura 1); observa-se ao longo do eixo 1 uma possível indicação de correlação da distribuição da fauna de abelhas sem ferrão com o gradiente latitudinal. As localidades da Mata Atlântica do Nordeste (exceto duas do sul da Bahia) estão concentradas na porção esquerda do gráfico (menores valores no eixo) e as localidades do Sudeste e Sul no centro na porção direita, com a localidade mais ao sul do gradiente ocupando o extremo direito do gráfico. Os pontos correspondentes às localidades não estão dispostos ordenadamente configurando um gradiente, apesar das localidades do Nordeste estarem agrupadas à esquerda do eixo 1 , as localidades mais ao norte (pontos 1,2 e 3 ) da região estão mais próximas às localidades das regiões Sudeste/Sul do que aquelas ao sul da Região Nordeste (pontos 4, 5). O segundo eixo sugere a existência de outros fatores determinando a composição de espécies além do gradiente per si; nota-se que os pontos de Ilhéus, Porto Seguro (BA) e Linhares (ES), respectivamente L6, L7 e L8, têm valores altos e positivos para o eixo 2. Destaca-se um elevado número de espécies únicas a uma 
localidade ou duas (13 e 5 espécies, respectivamente). A principal espécie associada às localidades ao sul de Santa Teresa (ES) é Plebeia remota. Para as localidades do norte da Mata Atlântica, Friesemellita francoi pode ser uma indicadora, apesar de ter sido registrada em apenas duas áreas. Distribuições de outras espécies que merecem destaque são a de Partamona criptica, que está presente apenas nas localidades da Bahia e do Espírito Santo e de Paratrigona subnuda, que agrupa as localidades do Rio de Janeiro e Salesópolis (SP).

Resultados preliminares da análise da distribuição da fauna de formigas amostradas pelo Projeto indicam com mais segurança o padrão latitudinal, que recebe ainda influência do gradiente altitudinal (Silva et al. 2007). Para as abelhas, a dispersão dos pontos é relativamente alta, o que pode ser resultado da amostragem ter sido insuficiente. Espécies com registro em apenas uma área devem, muito provavelmente, estar presentes em outras localidades, como Plebeia saiqui que deve ocorrer em outras áreas do Sudeste, e Lestrimellita tropica, que já foi registrada até o Ceará (Marchi \& Melo 2006). Além disso, gêneros comumente registrados em áreas de Floresta Atlântica como Cephalotrigona, Geotrigona, Nannotrigona e Tetragona não foram capturados nesta amostragem.

Relativamente poucos levantamentos de abelhas foram realizados em áreas de Floresta Ombrófila Densa em comparação a ambientes com vegetação aberta. Em Morretes (PR), foi amostrada uma localidade do Instituto Agronômico do Paraná por Barbola (2000), sendo catalogadas 111 espécies de 47 gêneros. Wilms (1995) amostrou extensivamente a Estação Ecológica de Boracéia, município de Salesópolis, São Paulo, encontrando 253 espécies de abelhas de 84 gêneros. Ramalho (1995) amostrou área na capital do Estado de São Paulo, encontrando 169 espécies de 56 gêneros. Estes números de espécies são relativamente elevados quando comparados com resultados de inventários conduzidos em áreas abertas (Figura 3 de Pinheiro-Machado 2002). Ao contrário, o presente inventariamento coletou números relativamente muito baixos de espécies nestas mesmas áreas, devido às técnicas empregadas.

Reunindo os dados dos diferentes levantamentos no domínio da Mata Atlântica é possível encontrar espécies que jamais foram coletadas em levantamentos em outros domínios, como Ariphanarthra palpalis Moure, 1951, Augochlorodes turrifaciens Moure, 1958 e Dithygater seabrai Moure \& Michener, 1955. Ainda, alguns grupos mostraram-se extremamente ricos em comparação à fauna registrada em estudos em outros ambientes, como Paracolletini, Meliponina, Euglossina e gêneros cleptoparasitas como Trophocleptria (Nomadini), Microsphecodes e Ptilocleptis (Halictini). Estes grupos e espécies em conjunto podem ajudar a caracterizar a fauna da Mata Atlântica, mas no presente trabalho foram registrados apenas Ariphanarthra e o elevado número relativo de Meliponina e de cleptoparasitas.

Ariphanarthra pertence a um grupo de gêneros de Augochlorini que inclui espécies com distribuições restritas ou claramente associadas à Mata Atlântica (Engel 2000). Outro exemplo deste grupo é Micrommation larocai Moure, 1969, que tem sido coletada exclusivamente em localidades da Mata Atlântica do Estado do Paraná, porém até agora jamais em levantamentos. Vale destacar que registramos exemplares que não puderam ser identificados, mas que pertencem a este grupo (Tabela 2).

A Mata Atlântica, ou componente Atlântico da região Neotropical, é relacionado ao sudeste Amazônico ou sua biota é relacionada a clados de ampla distribuição Neotropical, mas partições internas ao componente não são formalmente reconhecidas (p. ex. Camargo \& Pedro 2003). Estudos históricos sobre estas partições serão de grande valia para o entendimento da formação da melissofauna Atlântica. Quanto ao inventário de fauna, os estados ao norte de São Paulo apresentam áreas de especial interesse, pois não foram sujeitos a nenhuma amostragem quantitativa sistematizada até o momento.

\section{Agradecimentos}

Aos melitólogos que identificaram parte do material, Dra. Danúncia Urban (Anthidiini, Eucerini e Anthrenoides), Dr. João M. F. Camargo (Meliponina), Dr. Antonio J. C. Aguiar (Tapinotaspidini) e MSc. Luiz Roberto Faria Jr (Euglossina) pelo auxílio nas identificações do material; a todos os idealizadores e coletores do projeto, à Dra. Angélica M. P. Dias pelo auxílio na descrição dos procedimentos de coleta, ao Dr. Rogério R. Silva pelo auxílio com a bibliografia, sugestões de análise estatística e revisão do texto, à Dra. Eliana M. Cancello pela revisão final do manuscrito e à Biól. Nicolle V. Sydney pela leitura crítica do manuscrito. Este trabalho foi parcialmente financiado pela Fundação de Amparo à Pesquisa do Estado de São Paulo (FAPESP) no âmbito do Programa BIOTA/FAPESP - O Instituto Virtual da Biodiversidade (www.biotasp.org.br).

\section{Referências Bibliográficas}

AGNELLO, S. 2007. Composição, estrutura e comunidade de aves da Mata Atlântica no Parque Estadual da Serra do Mar - Núcleo Cubatão, São Paulo. Dissertação de Mestrado, Escola Superior de Agricultura "Luiz de Queiroz", Universidade de São Paulo, São Paulo.

AMORIM, D.S. \& PIRES, M.R.S. 1996. Neotropical biogeography and a method for maximum biodiversity estimation, p. 183-219. In: BICUDO, C.E.M. \& N.A. MENEZES. Biodiversity in Brazil: A First approach. CNPq, São Paulo.

BARBOLA, I.F. 2000. Biocenótica de Apoidea (Hymenoptera) de uma área restrita da Floresta Atlântica, Morretes, Paraná, Brasil, e aspectos da ecologia da polinização de Stachytarpheta maximiliani (Verbenaceae). Tese de Doutorado, Universidade Federal do Paraná, Paraná.

BROWN, J. \& LOMOLINO, M. 1998. Biogeography. Sinauer Associates, Sunderland, Massachusetts.

CAMARGO, J.M.F. \& PEDRO, S.R.M. 2003. Meliponini Neotropicais: O gênero Partamona Schwarz, 1939 (Hymenoptera, Apidae, Apinae): bionomia e biogeografia. Rev. Bras. Entomol. 47(3):311-372.

CANCELLO, E.M. 2006. Termite distribution in South America and its life histories: the data from Atlantic Forest survey and from MZUSP collection. In Annals of XV Internacional Congress of Iussi - International Union for the Study of Social Insects, Washington, DC, p. 149-150.

CANCELLO, E.M., REIS, Y.T., VASCONCELLO, A. \& OLIVEIRA, L.C. 2006. Riqueza de espécies de térmitas (Isoptera) ao longo de um gradiente latitudinal na Mata Atlântica, Brasil. In: Anais do XXI Congresso Brasileiro de Entomologia: da Academia à transferência de tecnologia, Recife, Pernambuco.

CONSERVATION INTERNATIONAL DO BRASIL, FUNDAÇÃO SOS MATA ATLÂNTICA, FUNDAÇÃO BIODIVERSITAS, INSITITUTO DE PESQUISAS ECOLÓGICAS, SECRETARIA DO MEIO AMBIENTE DO ESTADO DE SÃO PAULO, SEMAD/INSTITUTO ESTADUAL DE FLORESTAS - MG. 2000. Avaliação e ações prioritárias para a conservação da biodiversidade da Floresta Atlântica e Campos Sulinos. MMA/SBF, Brasilia.

COSTA, L.P., LEITE, Y.L.R., FONSECA, G.A.B., FONSECA, M.T. 2000. Biogeography of South American Forest Mammals: Endemism and Diversity in the Atlantic Forest. Biotropica 32:872-881.

ENGEL, M.S. 2000. Classification of the bee tribe Augochlorini (Hymenoptera: Halictidae). Bull. Am. Mus. Nat. His. 250:1-89.

FONSECA, G.A.B., HERMANN, G., LEITE, Y.L.R., MITTENMEIER, R. A., RYLANDS, A. B., PATTON, J. L. 1996. Lista anotada dos mamíferos do Brasil. Occas. Pap. Cons. Biol. 4:1-38.

FRASER, S.E.M., DYTHAM, C. \& MAYHEW, P.J. 2008. The effectiveness and optimal use of Malaise traps for monitoring parasitoid wasps. Ins. Cons. Div. 1:22-31. doi: 10.1111/j.1752-4598.2007.00003.x.

GASTON, K.J. 2000. Global patterns in biodiversity. Nature 405:220-227.

JANZEN, D.H. 1981. The peak in North american ichneumonid species richness lies between $30^{\circ}$ and $42^{\circ} \mathrm{N}$. Ecology 62:532-537. 
KRUG, C. \& ALVES-DOS-SANTOS, I. 2008. O uso de diferentes métodos para amostragem da fauna de abelhas (Hymenoptera: Apoidea), um estudo em Floresta Ombrófila Mista em Santa Catarina. Neo. Entomol. 37(3):265-278.

LAMBAIS, M.R., CROWLEY, D.E., CURY, J.C., BULL, R.C. \& RODRIGUES, R.R. 2006. Bacterial diversity in tree canopies of the Atlanctic Forest. Science 312:191.

LAROCA, S. 1974. Estudo feno-ecológico em Apoidea do litoral e primeiro planalto paranaense. Dissertação de Mestrado, Universidade Federal do Paraná, Paraná.

LONGINO, J.T. \& COLWELL, R.K. 1997. Biodiversity assessment using structured inventory: Capturing the ant fauna of a lowland tropical rainforest. Ecol. Appl. 7:1263-1277.

MARCHI, P., MELO, G.A.R. 2006. Revisão taxonômica das espécies brasileiras de abelhas do gênero Lestrimelitta Friese (Hymenoptera, Apidae, Meliponina). Rev. Bras. Entomol. 50(1):6-30.

MICHENER, C.D. 1979. The biogeography of the bees. Ann. Mo. Bot. Gard. 66:277-374.

MORELLATO, L.P.C \& HADDAD, C.F.B. 2000. Introduction: the Brazilian Atlantic Forest. Biotropica 32(4b):786-792.

MYERS, N., MITTERMEIER, R.A., MITTENMEIER, C.G., FONSECA, G.A.B. \& KENT. J. 2000. Biodiversity hotspots for conservation priorities. Nature 403:853-858.

OKSANEN, J., KINDT, R., LEGENDRE, P., O'HARA, B., STEVENS, M.H.H. 2007. Vegan: Community Ecology Package. R package version 1.8-8. http://cran.r-project.org/, http://r-forge.r-project.org/projects/ vegan/

OLIVEIRA-FILHO, A.T. \& FONTES, M.A. 2000. Patterns of floristic differentiation among Atlantic Forests in southeastern Brazil and the influence of climate. Biotropica 32(4b):793-810.

PINHEIRO-MACHADO, C. 2002. Brazilian bee biodiversity: what has been done and what is to be done. In Anais do V Encontro Sobre Abelhas, Universidade de São Paulo, Ribeirão Preto.

RAMALHO, M. 1995. A diversidade de abelhas (Apoidea, Hymenoptera) em um remanescente de Floresta Atlântica, em São Paulo. Tese de Doutorado, Universidade de São Paulo, São Paulo.
ROULSTON, T.H., SMITH, S.A., BREWSTER, A.L. 2007. A comparision of pan trap and intensive net sampling techniques for documenting a bee (Hymenoptera: Apiformes) fauna. J. Kansas Ent. Soc. 80(2):179-181.

SAKAGAMI, S. F., LAROCA, S. \& MOURE, J. S. 1967. Wild bee biocoenotics in São José do Pinhais (PR), South Brazil. Preliminary report. Journal of the Faculty of Science, Hokkaido University [Series VI, Zoology] 16:253-291.

SCHWARTZ-FILHO, D.L. \& LAROCA, S. 1999. A Comunidade de abelhas silvestres (Hymenoptera, Apoidea) da Ilha das Cobras (Paraná, Brasil): aspectos ecológicos e biogeográficos. Acta Biol. Par. 28:18-101.

SILVA, J.M.C., SOUZA, M.C., CASTELLETTI, C.H.M. 2004. Areas of endemism for passerine birds in the Atlantic forest, South America. Global Ecol. Biogeogr. 13:85-92.

SILVA, R.R., BRANDÃO, C.R.F. \& SCOTT-SANTOS, C.P. 2007. Riqueza, composição e caracterização do espaço morfológico da fauna de formigas de serrapilheira ao longo da Mata Atlântica. Biológico 62(2):289-290.

SOUZA, L. \& CAMPOS, M.J.O. 2008. Composition and diversity of bees (Hymenoptera) attracted by Moericke traps in ann agricultural area in Rio Claro, state of São Paulo, Brasil. Iheringia 98(2):236-243.

STEINER, J., HARTER-MARQUES, B., ZILLIKENS, A., FEJA, E. P. 2006 Bees of Santa Catarina Island, Brazil: a first survey and checklist (Insecta: Apoidea). Zootaxa 1220:1-18.

TER BRAAK, C.J.F. 1995. Ordination. Data Analysis in Community and Landscape Ecology (Jongman, R.H.G., Ter Braak, C.J.F. \& Van Torgeren, O.F.R., eds), p. 91-173. Cambridge University Press, Chicago.

WILLIG, M.R., KAUFMAN, D.M., STEVENS, R.D. 2003. Latitudinal gradients of biodiversity: pattern, process, scale, and synthesis. Annu. Rev. Ecol. Syst. 34:273-309.

WILMS, W. 1995. Die Bienenfauna im Küstenregenwald Brasiliens und ihre Beziehungen zu Blütenplanzen: Fallstudie Boracéia, São Paulo. Tese de Doutorado, Universidade de São Paulo, São Paulo.

Recebido em 15/06/08

Versão reformulada recebida em 08/10/08

Publicado em 23/10/08 